\title{
Land's Experiments in Color Vision and the Mathematical Structure of our Sense-Perception*
}

\author{
Seizo OHE \\ Nihon University, Tokyo
}

I

Since Mr. Edwin H. Land published the results of his interesting experiments in color vision three years ago [2], much or too much has been said about it [6]. It has aroused quite a sensation among opticians and T. V. engineers on the one hand and a hot debate in academic circles on the other. One of the representative types of criticism involved could be summarized as follows: the result which Land obtained through a series of elaborate experiments, namely "the color-image synthesis with two unorthodox primaries" can well be confirmed experimentally, but there is nothing new in it. The whole phenomenon, far from being "natural", is rather one of the most special cases of color vision and can adequately be explained in terms of old familiar theories of color, such as "adaptation", "contrast", "induction", etc. I do not intend to go into the details of this many-sided controversy, but simply to make my stand clear, first giving some necessary information on the topic.

A pair of positive black-and-white slides, which are made from a pair of negatives photographed of one and the same scene through a red and a green filter respectively, are projected with again a pair of longer and shorter wavelengths respectively, and their images are to be superposed on a white screen. If we choose e.g. red light for longer-wavelength projection and white light for shorterwavelength projection, the two images, of which one is red-washed and the other black-and-white, will produce, when well superposed on the screen, a picture with a surprising gamut of colors, instead of a simple pink-washed one, as it is supposed to be according to the classical theory of color mixture. (This type of Land's experiment will hereafter be referred to as Exp. I.) Thereby, the choice of wavelengths for projection is open to the extent that even within the yellow band of the color spectrum, e.g. orangish-yellow (around $599 \mathrm{~m} \mu$ ) and greenish-yellow (around $579 \mathrm{~m} \mu$ ) can be chosen for longer-and shorter-wavelength projection respectively, although hues at both ends of the color spectrum, red and violet approximately, can only be used as longer and shorter wavelengths respectively, and some

* This paper is essentially based upon another one which I read at the 1960 International Congress for Logic, Methodology and Philosophy of Science, Stanford, California, U.S.A.: "The Multiple Structure of our External Knowledge and Mr. Land's New Experiment in Color Vision". I feel much indebted to the encouragements and criticisms given either in the discussion there or later in letters, and also to many helpful talks which I have since had with Japanese specialists in this field. 
other limitations occur also in the middle part of the spectrum.

Land further reports a very interesting experiment (referred to hereafter as Exp. II). In place of a pair of picture slides in Exp. I, he simply used two stepwise graded black-and-white slides of exactly the same sort, one vertically placed in the red projector, the other horizontally in the white projector, or vice versa. The result was only a graded pink wash, in striking contrast with the colorful result of Exp. I. Attributing this result rather naively to the artificial regularity of step wedges and to the lack of that randomness which characterizes a natural image, Land concludes: "The colors in a natural image are determined by the relative balance of long and short wavelengths over the entire scene, assuming that the relationship changes in a somewhat random way from point to point. Within broad limits, the actual values of the wavelengths make no difference, nor does the over-all available brightness of each" [2].

To this conclusion, however, a fundamental revision now seems to be necessary.

In the meantime, Mr. Arthur Karp of Cambridge University has succeeded in producing a surprisingly beautiful gamut of colors by ingeniously modifying Exp. II. He simply replaced a pair of step-wedge slides in Exp. II by another pair which are "overlaid by either a black or a white network to form detached squares against a background" [1]. With the black network, the result was monochromatic, as in the original Exp. II, but with the white network (Fig. I-a, b) bluish-green hues drammatically appeared, as indicated in Fig. I-c* for the case with a red filter before Fig. I-b slide and no filter before Fig. I-a slide.

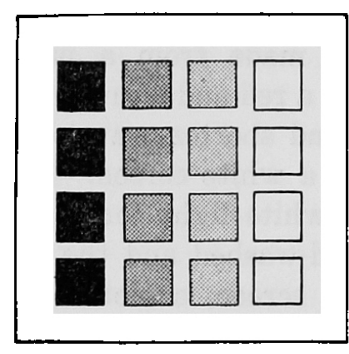

a.

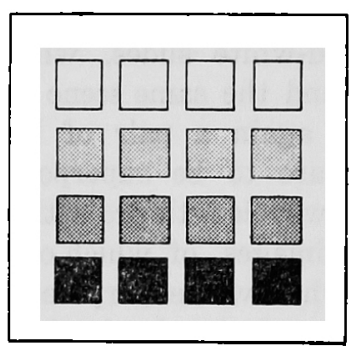

b.

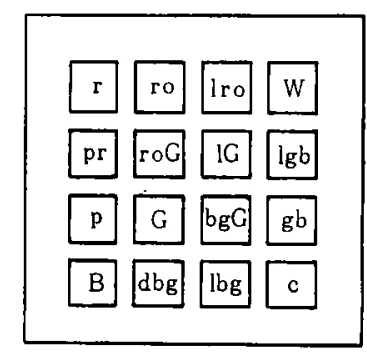

c.

Karp further found that points of the network or back-ground were pink when viewed in isolation through a small-aperatured telescope, but in context

* This experiment is very easy to perform even for an amateur experimenter like the present author. Mr. H. Terakado prepared the necessary slides. Fig. I is the result of our common work, though it is essentially the same as Fig. I (c) in Mr. Karp's report [1]. 
white, and that the bottom right-hand square was white in isolation, but cyanthe complementary of red-in context. With projection light of various other colors, corresponding results always ensued. [1]

Seen in the light of these new experimental findings, it can no longer be the irregularity of natural-image composition that really counts in Exp. I, and the whole phenomenon now seems to be reduced to a special, complicated case of such well-known, phenomenal color vision as "colored after-image", "colored shadow", etc. In this sense Land undoubtedly went too far in some of his scientific assertions, but what he found by his ingenious experiments seems, for the most part, to have since been confirmed. Assuming the discovered phenomena be primarily of a psychological order, we only see, how strongly our actual color vision in daily surroundings is psychologically affected. This is not at all surprising, if we think of the fact that the psychological system of our perceptual colors, the "color circle", has a structure entirely different from the physical system of visible lights, the prismatic "color band".

In this connection, it may well be remembered that this closed system of our perceptual hues can be shown to have a group-like structure in the mathematical sense, having color mixture as an additive operation, neutral white as the identity element, and complementary hues as the inverse elements. [3]

\section{II}

In spite of all that has rightly been said about the psychological platitude of Land's experiments, there still remains another aspect to be carefully examined. I do not mean the yet unclarified physiological mechanism behind all those psychological facts of our actual color vision, but rather his physico-technical approach to the problem. How to attain sufficient color effect by the simplest means? What can be the physical regularity which theoretically supports the possible procedure? These are the questions that Land has raised and solved in his way.

It need hardly be said that I do not intend to evaluate his technique of colorimage synthesis as such. What interests me is merely the theoretical background thereof. To repeat, quoting partly Land himself: "Colors in images arise not from the choice of wavelength but from the interplay of longer and shorter wavelengths over the entire scene, depending upon a varying balance between longer and shorter wavelengths in its various parts" [2]. The independence of wavelength and color is in itself not so surprising as it might sound in the above wording. It is rather an established fact, as far as our actual, in many ways psychologically affected color vision is concerned. More remarkable, however, is the fact that colors, at least in some cases, seem to depend upon certain measurable patterns of balance between longer and shorter wavelengths, which somehow remain constant throughout the pretty wide range of the color spectrum, though not without some subtle restrictions.

Right at this point, however, an analogous, familiar situation with our spoken vowels springs to our eyes almost inevitably. We know that a vowel can be pronounced practically at every pitch of the human voice, and that every vowel 
shows two characteristic rosonance bars, called 1st and 2nd formants in the ascending order of c. p.s., when it is analyzed by sonagraph. The pattern is roughly as in Fig. II-a.

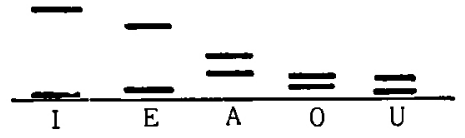

a. Sona-gram patterns of principal vowels, representing only the two strongest resonance bars, from "Visible Speech" by Potter-Kopp-Green [4]

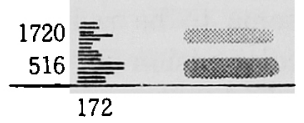

i. Lower pitch:

b. Resonance analysis and sona-graph of Japanese E from "Beautiful Vocalization" (Jap.) by R. Taguti [5]

Fig. II

These bars represent simply the two strongest rosonance components and such a resonance-bar pattern remains nearly the same all along with the corresponding vowel characteristic throughout the entire pitch-range of the human voice. When we now consider the set of all possible pitch-shifts (i.e. raises and drops) in vowel pronunciation as a group of transformations in the sense that every transformation has an "inverse" also belonging to the set (i.e. for a raise a drop of the same size), and the resultant of any two consecutive transformations within the threshold of perceptibility also belongs to the set, then a vowel characteristic with its corresponding resonance-bar pattern will be the "invariant" of this transformation group, as is indicated in Fig. II-b for Japanese vowel E. That means, to borrow Land's wording, vowels in the human voice arise essentially from the interplay of its two main rosonance components, a pair of lower and higher frequencies. Translated again into the color phenomena of Land, a color can now be regarded as the "invariant" under transformations of all possible shifts of the balancepoint between longer and shorter wavelengths throughout the middle range of the visible spectrum, which might form a kind of group-like structure analogous to that of all possible pitch-shifts in vowel pronunciation, though everything here is as yet far more limited and uncertain.

On the other hand, the system of our spoken vowels itself can also be interpreted as a group-like structure of its own, while, according to $\mathrm{W}$. Köhler, the "physical vowels", i.e. U-, O-, A-, E-, I- vowel qualities of pure physical tones, can experimentally be assigned to the frequencies $\mathrm{c}^{1}, \mathrm{c}^{2}, \mathrm{c}^{3}, \mathrm{c}^{4}, \mathrm{c}^{5}$ in the ascending order of pure aerial vibrations. [3]

Again, it may be worth noting that the relation between this "band of physical vowels" and the "vowel triangle" of the human voice has a striking similarity to the relations between the physical "color band" and the psychological "color circle", although they actually show a sharp contrast as regards the perceptual values they represent in their respective kinds of senses. 


\section{III}

From all that precedes, it is permissible to conclude that, firstly, in the world of our visual and auditory perception there seems to be prevalent a kind of wellordered, closed structure essentially akin to the mathematical conception of group, the physio-psychological root of which, as it were, might be the former, and that, secondly, this very group-like structure with its characteristic closedness and order strongly suggests a certain independent meaning in our perceptual cognition of the conceptual frame of our mathematical science of nature, as once Goethe so tenaciously fought for against Newton.

(Received Nov. 29, 1961)

\section{References}

[1] Karp, A.: Colour-image synthesis with two unorthodox primaries; Nature, 184, 710 (1959)

[2] Land, E. H.: Experiments in color vision; Sci. Amer., 84 (May 1959)

[3] Ohe, S.: Application of mathematical group concept to human perceptual systems, visual and auditory; Ann. of the J.A. P.S., 1, 2, 11 (1957)

[4] Potter-Kopp-Green: Visible Speech, New York, 1947, P. 60.

[5] Taguti, R.: Beautiful Vocalization (Jap.), Tokyo, 1958, P. 52.

[6 ] Wallis, G. L.: Land! Land!; Psychol. Bull., 59, 1, 30 (1960) 